\title{
Functional fungal extracts from the Quorn fermentation co-product as novel partial egg white replacers
}

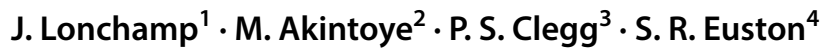

Received: 19 June 2019 / Revised: 6 September 2019 / Accepted: 19 October 2019 / Published online: 13 November 2019

(c) The Author(s) 2019

\begin{abstract}
The production of mycoprotein biomass by Marlow Foods for use in their meat alternative brand Quorn is a potential source of sustainable alternatives to functional ingredients of animal origin for the food industry. The conversion of this viscoelastic biomass into the Quorn meat-like texture relies on functional synergy with egg white (EW), effectively forming a fibre gel composite. In a previous study, we reported that an extract (retentate 100 or R100) obtained from the Quorn fermentation co-product (centrate) via ultrafiltration displayed good foaming, emulsifying, and rheological properties. This current study investigated if a possible similar synergy between EW and R100 could be exploited to partially replace EW as foaming and/ or gelling ingredient. The large hyphal structures characteristic of R100 solutions were observed in EW-R100 mixtures, while EW-R100 gels showed dense networks of entangled hyphal aggregates and filaments. R100 foams prepared by frothing proved less stable than EW ones; however, a 75/25 w/w EW-R100 mixture displayed a similar foam stability to EW. Simlarly, R100 hydrogels proved less viscoelastic than EW ones; however, the viscoelasticity of gels prepared with 50/50 w/w and 75/25 w/w EW-R 100 proved similar to those of EW gels, while 75/25 w/w EW-R100 gels displayed similar hardness to EW ones. Both results highlighted a functional synergy between the R100 material and EW proteins. In parallel tensiometry measurements highlighted the presence of surface-active material in EW-R100 mixtures contributing to their high foaming properties. These results highlighted the potential of functional extracts from the Quorn fermentation process for partial EW replacement as foaming and gelling agent, and the complex nature of the functional profile of EW-R100 mixtures, with contributions reported for both hyphal structures and surface-active material.
\end{abstract}

J. Lonchamp

JLonchamp@qmu.ac.uk

1 School of Health Sciences, Queen Margaret University Drive, Queen Margaret University, Edinburgh EH21 6UU, UK

2 Quorn Foods, Station Road, Stokesley TS9 7BR, UK

3 School of Physics \& Astronomy, The University of Edinburgh, Peter Guthrie Tait Road, Edinburgh EH9 3FD, UK

4 Institute of Mechanical, Process \& Energy Engineering, School of Engineering and Physical Sciences, Heriot-Watt University, Edinburgh EH14 4AS, UK 


\section{Graphic abstract}
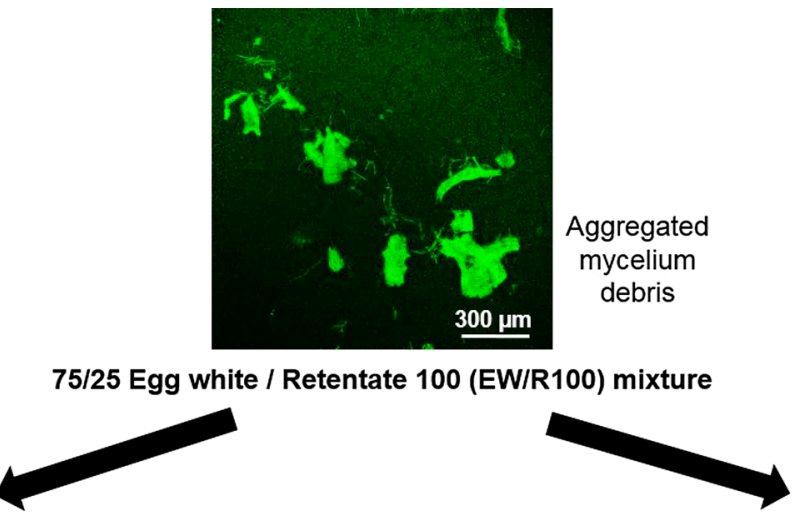

75/25 EW / R100 foam

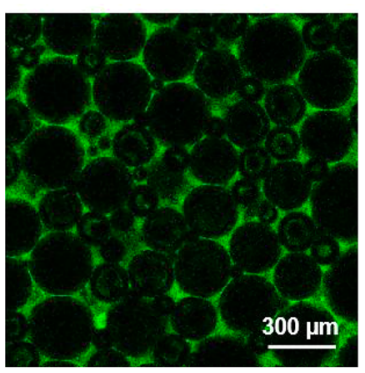

Mycelium network

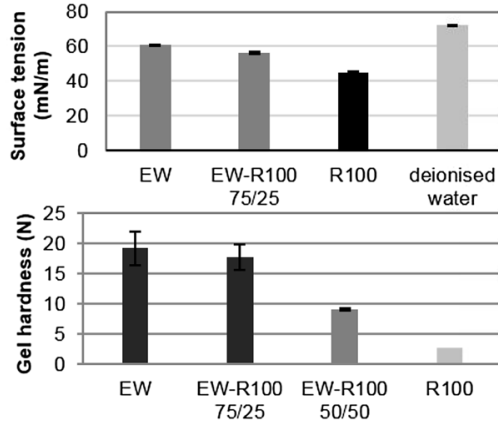

75/25 EW / R100 hydrogel

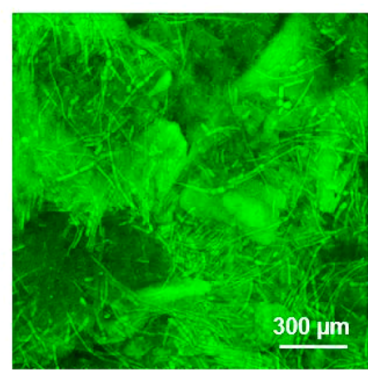

Aggregated structures

Keywords Quorn $\cdot$ Co-product $\cdot$ Centrate $\cdot$ Egg $\cdot$ White $\cdot$ Replacement

\section{Introduction}

Due to its excellent foaming properties, egg white is widely used in desserts, cakes, biscuits, and many aerated prepared dishes including soufflés and mousses. Egg white also displays excellent gelling properties used for various cooking or baking applications. However, due to the high environmental costs and market volatility of animal-derived functional ingredients including milk and egg proteins, the food industry is looking for sustainable alternatives [1]. One of the strategies employed consists in screening unexploited co-product streams from the food industry for extraction of potential functional alternatives. In this context, the production of mycoprotein by Marlow Foods for use in their meat alternative product Quorn is a potential source of sustainable functional ingredients. The term mycoprotein refers to the high-protein biomass produced by fermentation of the fungus Fusarium venenatum A3/5 (ATCC PTA-2684) by Marlow Foods, which forms the basis of their Quorn brand products. Mycoprotein contains all essential amino acids [2] and the net protein utilisation value of Fusarium venenatum mycoprotein is comparable to that of milk [3]. Moreover, the fermentation of starch into protein by Fusarium venenatum results in $90 \%$ lower emission of greenhouse gases and benefits in terms of land and water footprints in comparison with beef products [4].

Following fermentation, the broth undergoes an RNAreduction step during which it is first heat-shocked above $68{ }^{\circ} \mathrm{C}$ for $30-45 \mathrm{~min}$, then further heated at $90{ }^{\circ} \mathrm{C}$, and finally centrifuged [5]. This heat shock step stops growth, disrupts ribosomes, and activates endogenous RNAases which break down cellular RNA to nucleotides [5]. The resulting solid deposit (mycoprotein biomass) is then processed into a dough ready for conversion to the meat-like texture characteristic of Quorn foods. However, the heatshock RNA-reduction step also induces diffusion of a fraction of the cell components through the cell wall [5]. The liquid supernatant (centrate) generated by the subsequent centrifugation step thus contains residual hyphal biomass, carbohydrates, nucleotides, and proteins as well as the residues of the fermentation feedstock, and is currently an unexploited co-product stream.

Following centrifugation, the viscoelastic mycoprotein biomass is mixed with egg albumen and the material is discharged into industrial block formers. The pressure exerted allied with the flow characteristics of the mixed material result in the introduction of laminations or layers which can be considered as textural precursors for the 
final meat-like texture [6]. The mix is heated to $90{ }^{\circ} \mathrm{C}$ then chilled and frozen to approximately $-10{ }^{\circ} \mathrm{C}$. This controlled freezing step results in an entangled mass of mycoprotein hyphae with gelled albumen protein within the interstitial space, a system described as fibre gel composite which confers a meat-like texture to the products [6].

Egg white is a complex system of proteins with different physicochemical properties. Lysozyme $(14.3 \mathrm{kDa})$ is a very basic protein with an isoelectric point at $\mathrm{pH} 10.5$, making it the only egg white protein that is positively charged in physiological conditions [7]. The high foaming properties of egg white could be due to a synergy in adsorption at the air/water interface between the positively charged lysozyme and other negatively charged proteins including ovalbumin and ovotransferrin, with intermolecular interactions occurring between the oppositely charged proteins after their unfolding, which stabilises the air bubbles [8-10]. Such synergy at the air/water interface has also been reported between lysozyme and whey proteins $[11,12]$, with intermolecular interactions reported in the bulk solution as well as at the air-water interface, resulting in higher foam stability due to the reduction of electrostatic repulsive interactions in the protein film.

In a previous study [13], we reported that an extract (retentate 100 or R100) obtained via a $100 \mathrm{kDa}$ ultrafiltration of the centrate displayed good foaming stability, emulsifying, and rheological properties (viscosity, viscoelasticity, and gelation). R100 solutions displayed high viscosity, while R100 solutions and hydrogels showed high viscoelasticity. R100 foams displayed high stability, while oil-inwater R100 emulsions showed small and stable oil droplet size distributions. Large hyphal aggregates were reported in R100 solutions and gels, correlating with their high viscosity and viscoelasticity. A dense hyphal network was observed in R100 foams and contributed to their stability. In parallel tensiometry measurements at the oil/water interface highlighted the presence of interfacially active molecules in R100 which formed a rigid film stabilising the oil droplets. A number of functional metabolites and proteins were identified in the centrate, including a ceratoplatanin protein, cell membrane constituents (phospholipids, sterols, glycosphingolipids, and sphingomyelins), cell wall constituents (chitin, chitosan, proteins), and guanine and guanine-based nucleosides and nucleotides. The current study investigated if a similar synergy to the one reported between EW and mycoprotein in the formation of the Quorn meat-like texture could be exploited between EW and R100 with a view to partially replacing EW as foaming and/or gelling ingredient. As part of this work, the functional profile (foaming and rheological properties) of a range of mixtures of EW and R100 was characterised.

\section{Materials and methods}

\section{Sample preparation}

Centrate samples were collected from the Marlow Foods fermenter site at Belasis, Billingham and frozen. Following thawing, the centrate underwent a $100 \mathrm{kDa}$ ultrafiltration step using a Vivaflow 200 crossflow cassette (Sartorius, UK) connected to a Masterflex Easy-Load peristaltic pump (Sartorius, UK). The resulting retentate 100 or R100 (composed of molecules larger than $100 \mathrm{kDa}$ ) was freeze-dried in a Super Modulyo unit (Edwards, UK). A commercial agglomerated egg white (EW) powder was used as control.

\section{Nitrogen content}

We previously reported that a wide range of functional molecules present in centrate and concentrated in the R100 fraction contained nitrogen, including fungal cell membrane and cell wall constituents such as phospholipids, glycosphingolipids, sphingomyelins, chitin, chitosan, and proteins [13]. In that regard, the nitrogen-containing material (NCM) content was measured using the Kjeldahl method [14] to provide a guideline for preparation of standardised sample quantities for functional tests. Sets of $0.1 \mathrm{~g}$ of EW and R100 were digested in concentrated sulphuric acid (92\%) using a Kjeltec Basic Digestion Unit 20 (Foss, UK) at $440{ }^{\circ} \mathrm{C}$ in the presence of a selenium catalyst. The digested samples were then distilled into boric acid using a Tecator Kjeltec 8100 Manual Distillation Unit (Foss, UK). Finally, the distilled samples were titrated using $0.1 \mathrm{~N}$ hydrochloric acid. The $\%$ nitrogen was calculated using the following formula:

$\%$ Nitrogen $=\frac{\text { Titration volume }(\mathrm{ml}) \times 14.007}{\text { Sample weight }(\mathrm{g}) \times 100}$.

The $\%$ nitrogen obtained was then multiplied by the general conversion factor of 6.25 recommended for mycoprotein biomass [15] to obtain a $\%$ nitrogen-containing material (NCM). Measurements were repeated three times. The functional profile of the four following samples was then assessed: EW control, R100, 75/25 EW-R100 (75\% $\mathrm{EW}+25 \% \mathrm{R} 100$ on $\mathrm{w} / \mathrm{w}$ basis for specified NCM content), and $50 / 50 \mathrm{EW}-\mathrm{R} 100(50 \% \mathrm{EW}+50 \% \mathrm{R} 100$ on w/w basis for specified NCM content).

\section{Gel electrophoresis}

Sodium dodecyl sulfate polyacrylamide gel electrophoresis (SDS-PAGE) was performed using a Mini-Protean Tetra Cell System with Mini-Protean TGX 4-20\% Tris-glycine precast gels (Bio-Rad Laboratories Ltd., UK) according to 
the methods of Laemmli [16] and Havea et al. [17] with minor modifications. One percent w/w nitrogen-containing material (NCM) solutions of the EW, R100 and 75/25 EW-R100 samples were prepared in deionised water and stirred for $1 \mathrm{~h}$. One set of each solution was sonicated for 3 min at $20 \mathrm{kHz}$ and 50\% amplitude using a Sonics VibraCell VCX-500 probe sonicator (Sonics, UK) to provide a reference for protein de-aggregation, while the other set was left untreated.

SDS-PAGE samples were prepared by mixing equal proportions of sample solution and Laemmli $2 \times$ concentrate sample buffer (Sigma Aldrich Ltd., UK) followed by heating at $70{ }^{\circ} \mathrm{C}$ for $10 \mathrm{~min}$. The See Blue Plus2 pre-stained standard (Life Technologies Ltd., UK) was used as molecular weight marker. The wells were loaded with $20 \mu \mathrm{l}$ of sample or $10 \mu \mathrm{L}$ of marker and the gels were run in Tris/Glycine/ SDS buffer (Bio-Rad Laboratories Ltd., UK) at $100 \mathrm{~V}$ for $1 \mathrm{~h}$. The gels were subsequently stained in a Coomassie brilliant blue solution (VWR Ltd., UK) for $1 \mathrm{~h}$ and destained overnight in the corresponding destaining solution (glacial acetic acid:methanol:deionised water 1:4:5). Gels were scanned using a ChemiDoc XRS + imaging system (Bio-Rad Laboratories Ltd., UK) and analysed using the associated Image Lab software.

\section{Rheological properties}

Viscosity and gelation measurements were performed using a Bohlin Gemini controlled stress rheometer (Malvern Instruments, UK) using cone-and-plate geometry. $10 \% \mathrm{w} / \mathrm{w}$ NCM solutions of EW, R100, 75/25 EW-R100, and 50/50 EW/R100 were prepared in deionised water and stirred for $2 \mathrm{~h}$. To assess the potential contribution of the R100 material to the EW gelling profile, EW solutions at $7.5 \%$ and $5 \% \mathrm{w} / \mathrm{w}$ NCM concentrations (matching the respective EW concentrations of the 75/25 EW-R100 and 50/50 EW/R100 samples) were also tested. Measurements were repeated three times.

Viscosity measurements were performed using a $4^{\circ} / 40 \mathrm{~mm}$ cone (gap $150 \mu \mathrm{m}$ ) at $20^{\circ} \mathrm{C}$. The instantaneous viscosity (Pa.s) was measured through a shear rate increase from 0.001 to $50 \mathrm{~s}^{-1}$. Prior to gelation tests, the linear viscoelasticity region of each sample was determined via oscillatory measurements of elastic and viscous moduli (G' and G') carried out at $1 \mathrm{~Hz}$ over a strain amplitude sweep ranging from 0.00005 to 50 . Gelation profiles were then assessed via small-amplitude oscillatory measurements using a $2 \% / 40 \mathrm{~mm}$ cone (gap $70 \mu \mathrm{m}$ ) with the applied strain chosen from within the linear viscoelastic region for each sample. The elastic and viscous moduli (G' and G') were measured through a temperature sweep test ranging from 40 to $90{ }^{\circ} \mathrm{C}$ in up-down mode ( $15 \mathrm{~min}$ up-sweep and $15 \mathrm{~min}$ down-sweep) and the oscillation frequency was $1 \mathrm{~Hz}$.

\section{Texturometry on hydrogels}

Ten milliliter solutions of $10 \% \mathrm{w} / \mathrm{w}$ NCM EW, R100, 75/25 EW-R100, and 50/50 EW/R100 were prepared in deionised water and stirred for $2 \mathrm{~h} .10 \mathrm{ml}$ solutions of $7.5 \%$ and $5 \% \mathrm{w} / \mathrm{w}$ NCM EW were also assessed as controls. The solutions were then heated for $1 \mathrm{~h}$ at $95^{\circ} \mathrm{C}$ in a water bath and cooled down at $4{ }^{\circ} \mathrm{C}$ for $3 \mathrm{~h}$. The resulting gels were tested for hardness using a BDO-FBO5.TS texture analyser (Zwick-Roell, UK) equipped with a $40 \mathrm{~mm}$ height $/ 20 \mathrm{~mm}$ bottom diameter cylindrical probe. A single compression test was performed at a $1 \mathrm{~mm} / \mathrm{sec}$ crosshead speed and $10 \mathrm{~mm}$ gel penetration depth. Measurements were replicated twice. The results were analysed using the associated testXpert 11.02 software (Zwick-Roell, UK).

\section{Foaming properties}

Two types of foaming methods (frothing and gas sparging) were carried out at room temperature $\left(20^{\circ} \mathrm{C}\right)$. Both foaming ability and stability were investigated using a frothing test. Fifteen gram solutions of $1 \% \mathrm{w} / \mathrm{w}$ NCM solutions of EW, R100, 75/25 EW-R100, and 50/50 EW/R100 were prepared in $50 \mathrm{ml}$ glass beakers, which corresponds to an initial volume of $18 \mathrm{~cm}^{3}(1.4 \mathrm{~cm}$ sample height and $4 \mathrm{~cm}$ beaker diameter), and stirred for $1 \mathrm{~h}$. To assess the potential contribution of the R100 material to the EW foaming profile, EW solutions at $0.75 \%$ and $0.5 \% \mathrm{w} / \mathrm{w} \mathrm{NCM}$ concentrations (matching the respective EW concentrations of the 75/25 EW-R100 and 50/50 EW/R100 samples) were also tested. The solutions were frothed for 1 min using a handheld whisk-type frother (Aerolatte, UK). The height of the resulting foam was measured immediately after whisking and every 10 min until collapse of the foam. The foaming ability was expressed as the initial height of the foam, while the foam stability was determined as the time needed for the foam to fully collapse. Measurements were repeated twice.

A gas-sparging test was also used according to Rudin [18] with minor modifications to assess the stability of foams prepared with EW, R100, and EW-R100 mixture solutions. $40 \mathrm{~g}$ solutions of $1 \% \mathrm{w} / \mathrm{w}$ NCM solutions of EW, R100, and $50 / 50 \mathrm{EW} / \mathrm{R} 100$ were stirred for $1 \mathrm{~h}$ and transferred to a column, which corresponds to an initial volume of $48 \mathrm{~cm}^{3}$ (3.8 cm sample height and $4 \mathrm{~cm}$ column diameter). A $0.5 \%$ w/w NCM EW was also tested as control. Gas sparging was carried out at constant flow rate of $\mathrm{CO}_{2}\left(100 \mathrm{~cm}^{3} / \mathrm{min}\right)$ until the foam reached the top indicator of the column (at $32.5 \mathrm{~cm}$ height from the bottom), which corresponds to a foam volume of $409 \mathrm{~cm}^{3}$. The foam stability was quantified as the time needed for the foam to collapse to the bottom indicator of the column. Measurements were repeated three times. 


\section{Surface tension}

One percent w/w NCM solutions of EW, R100, and 75/25 EW-R100 were serially diluted to obtain $0.5 \%, 0.25 \%$, and $0.1 \% \mathrm{w} / \mathrm{w}$ NCM solutions. The surface tension of the solutions was measured using the Du Nouy method with an EZ-Pi Plus tensiometer (Kibron, UK). Deionised water was used as control with a surface tension reading of $72 \mathrm{mN} / \mathrm{m}$. $1.5 \mathrm{ml}$ of solution was placed into a sterile cup holder. Triplicate measurements were carried out at $20^{\circ} \mathrm{C}$.

\section{Confocal microscopy}

EW, R100, 75/25 EW-R100, and 50/50 EW/R100 solutions, foams, and hydrogels were imaged using a Leica TCS2 confocal laser scanning microscope (Leica Microsystems, Germany). $1 \%$ and $10 \% \mathrm{w} / \mathrm{w}$ NCM solutions were prepared in deionised water supplemented with the fluorescent dye rhodamine B, respectively, stirred for $1 \mathrm{~h}$ and $2 \mathrm{~h}$, and imaged. Foams and hydrogels were prepared following the procedures described in the foaming and texturometry sections and imaged. The dye was excited at $514 \mathrm{~nm}$, the collection range was $600-700 \mathrm{~nm}$, and a $10 x / 0.25$ dry lens was used. Micrographs were recorded at a resolution of $512 \times 512$ pixels and analysed using the manufacturer's software (Leica Software Development Kit DM SDK version 4.2.1).

\section{Human/animal rights}

This article does not contain any studies with human or animal subjects.

\section{Results and discussion}

\section{Characterisation of EW-R100 mixtures}

The NCM content of the R100 and agglomerated EW samples were, respectively, $55.2 \%$ and $91.8 \%$ based on Kjeldahl measurements. The NCM content of the R100 fraction obtained was similar to the one measured in our previous study $(56.7 \%)$ [13].

As previously observed [13], large hyphal structures were present in R100 (Fig. 1c), resulting from the aggregation of centrate hyphal debris during their retention on the $100 \mathrm{kDa}$ ultrafiltration membrane. Hyphal structures were also observed in the 75/25 EW-R100 mixture (Fig. 1b). Fungal aggregate formation has been attributed to a combination of electrostatic interactions, hydrophobic interactions, and specific interactions between cell wall components including proteins and polysaccharides [19], and the influence of temperature on hyphal aggregation was previously reported for the filamentous fungus Rhizopus sp. [20]. The aggregation process observed could thus have resulted from the pressure exerted on the ultrafiltration membrane-retained material and/or from the temperature increase occurring during ultrafiltration.

To visualise both the concentrated EW protein bands and the fainter R100 ones, a high-contrast scan of the SDS-PAGE gel was produced (Fig. 2). The main egg white proteins were observed on the SDS-PAGE profile of the untreated agglomerated EW sample (Fig. 2), including ovalbumin $(45 \mathrm{kDa})$, ovotransferrin $(76-80 \mathrm{kDa})$, and lysozyme (14 $\mathrm{kDa})$. An additional band (A) was observed in untreated agglomerated EW, but disappeared following sonication or combination with untreated or sonicated R100. The molecular weight of band A matched with that of a lysozyme dimer $(28 \mathrm{kDa})$, which was in accordance with previous reports in which lysozyme was shown to dimerise when heated [21]. In
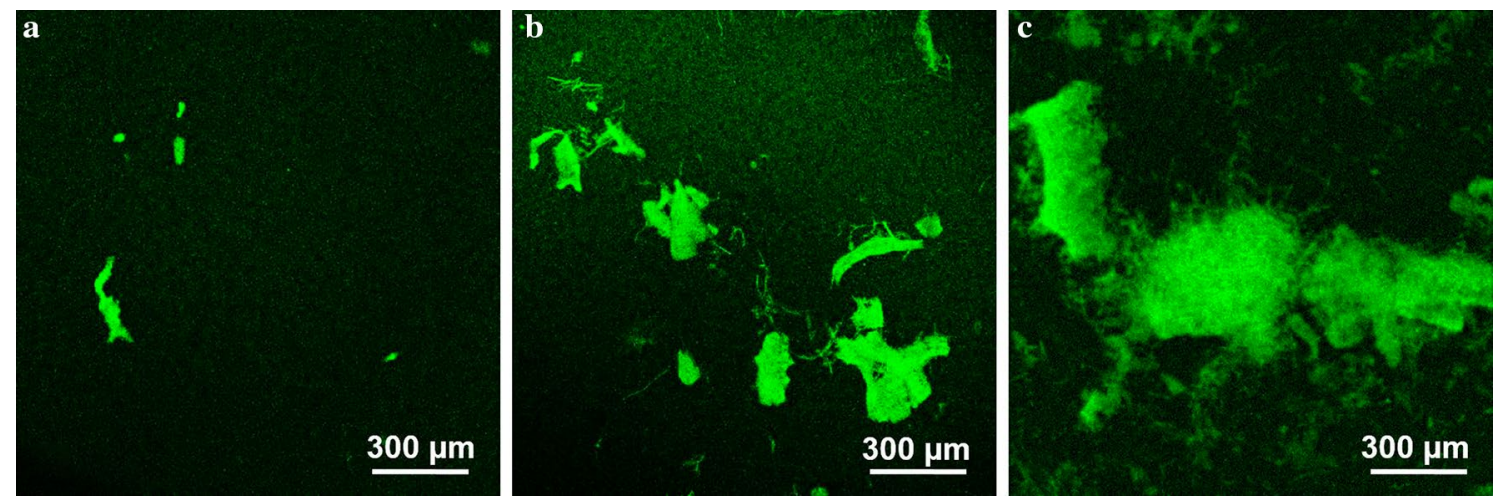

Fig. 1 Confocal microscopy of EW, R100, and 75/25 EW-R100 solutions (1\% w/w NCM concentration, rhodamine, magnification $\times 10)$. a EW, b 75/25 EW-R100, and c R100 


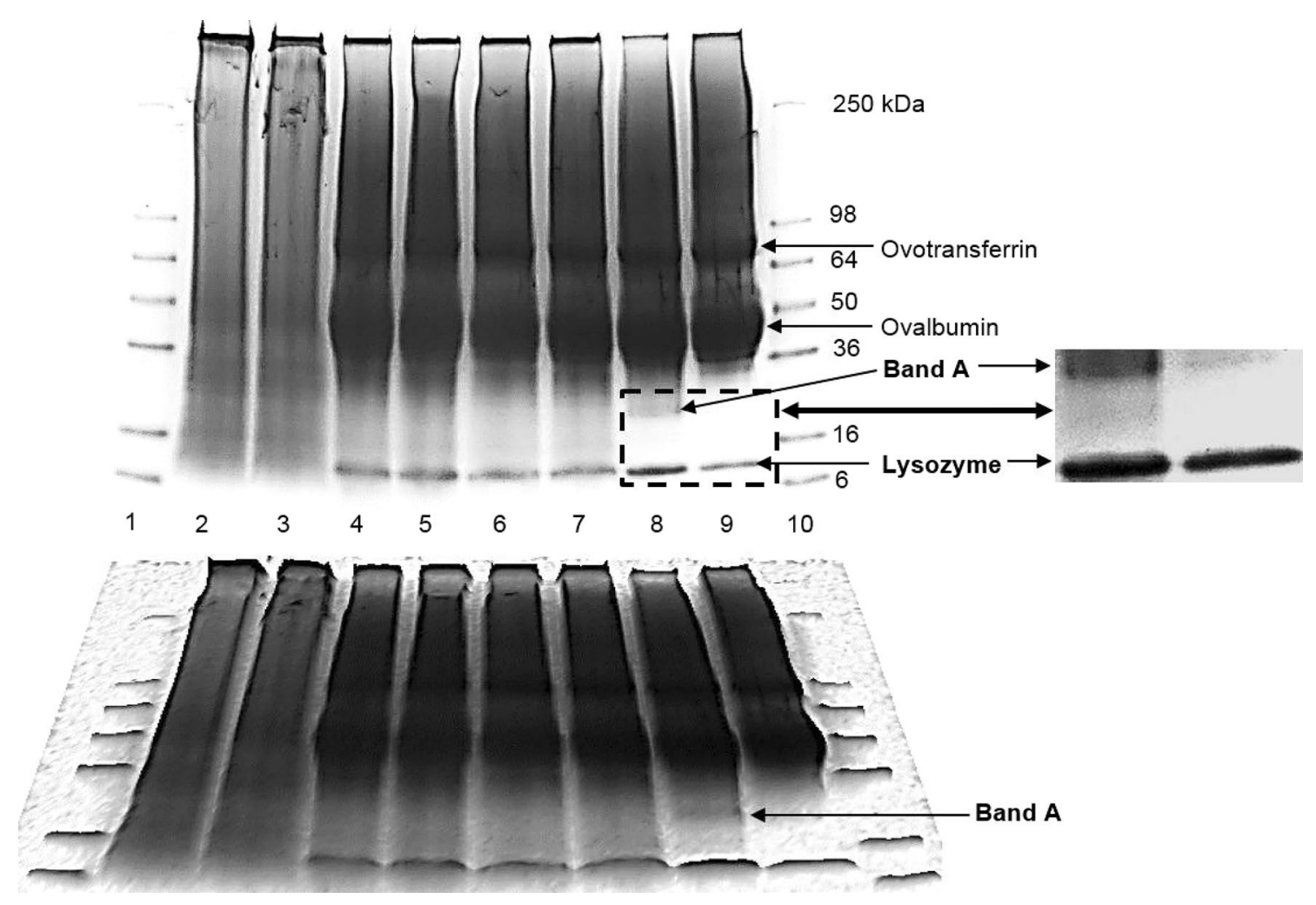

Fig. 2 SDS-PAGE of native and sonicated EW, R100, and EW-R100 solutions (1\% w/w NCM, original $\mathrm{pH}$, and high-contrast gel scan). (1) See blue marker, (2) untreated R100, (3) sonicated R100, (4, 5) untreated 75/25 EW-R100, (6, 7) sonicated 75/25 EW-R100, (8) untreated EW, (9) sonicated EW, and (10) see blue marker

Another explanation for the EW-R100 gel profile was the potential binding of monomeric and dimeric lysozyme forms to fungal cell walls. Lysozymes, also known as muramidases or $\mathrm{N}$-acetylmuramide glycanhydrolases, are a family of enzymes binding and catalysing the hydrolysis of $\beta-1-4$ glycosidic bonds within peptidoglycans contained in bacterial cell walls, resulting in antimicrobial effects [24]. Due to their chemical similitude with peptidoglycan (both type of polysaccharides contain $\beta-1-4$ linked $N$-acetylglucosamine units), chitin and chitosan contained in fungal cell walls have also been reported as viable binding and hydrolysis substrates for a number of lysozymes including egg white lysozyme $[25,26]$.

\section{Rheological properties of EW-R100 mixtures}

R100 and 50/50 EW-R100 solutions proved more viscous than EW ones at $10 \%$ w/w NCM (Fig. 3) due to the presence of the large hyphal structures observed in Fig. 1, which resisted flow. Similarly, a high concentration of hyphal aggregates was previously correlated with a decrease of the flow index for fermentation broths of the filamentous fungus Aspergillus terreus [27]. In parallel, our previous study [13] also showed the presence of compounds with known 


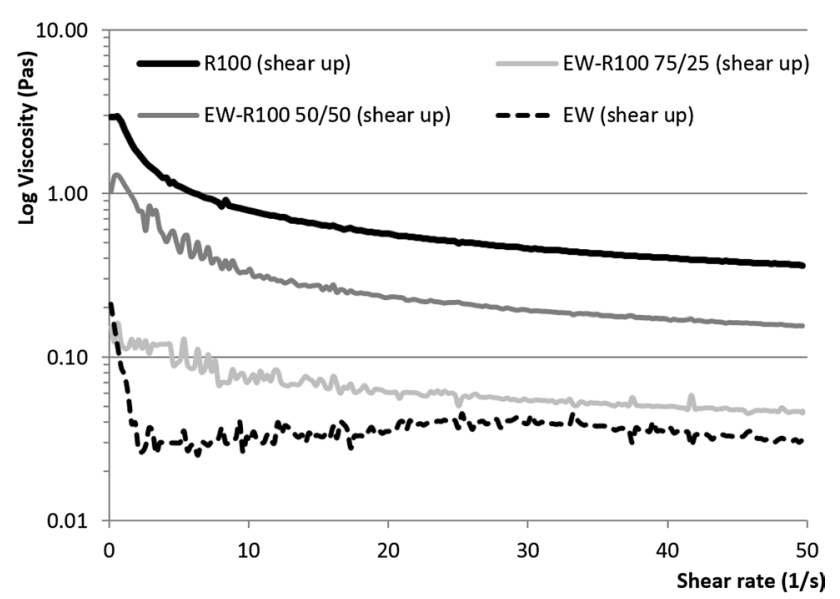

Fig. 3 Viscosity profiles of EW, R100, and EW-R100 mixtures during shear rate increase $(10 \% \mathrm{w} / \mathrm{w} \mathrm{NCM}$ concentration, original $\mathrm{pH}$, $n=3$ ) viscosifying properties in the centrate, including cell wall and membrane constituents which concentrated in the R100 fraction as part of the hyphal aggregates and contributed to the viscosity of EW-R100. The viscosifiers reported included chitin and chitosan [28, 29], inulin [30], galactan [31], nucleosides and nucleotides (including guanine-based compounds) and their derivatives [32], and sugar alcohols [33].

R100 and both 75/25 and 50/50 EW-R100 solutions showed higher viscous and elastic moduli than EW at $10 \%$ w/w NCM at $40{ }^{\circ} \mathrm{C}$ prior to gelation (Fig. 4, viscous moduli results not shown). 75/25 and 50/50 EW-R100 hydrogels displayed similar viscous and elastic moduli to EW ones and higher values than $7.5 \%$ and $5 \% \mathrm{EW}$ ones. The viscous and elastic moduli of R100 gels proved higher than $5 \% \mathrm{EW}$ ones, but lower than $7.5 \% \mathrm{EW}$ ones. The viscoelasticity of R100 gels was due to the very dense networks of entangled hyphal aggregates and filaments formed during gelation as observed in Fig. 5c. The entanglement of hyphal aggregates and filaments reported for EW-R100 gels was reminiscent of the microstructure of Quorn products, which is responsible for

Fig. 4 Gelation profiles (elastic modulus G') of EW-R100 mixtures $(10 \%, 7.5 \%$, or $5 \% \mathrm{w} / \mathrm{w}$ $\mathrm{NCM}$ concentration, original $\mathrm{pH}, n=3$ )
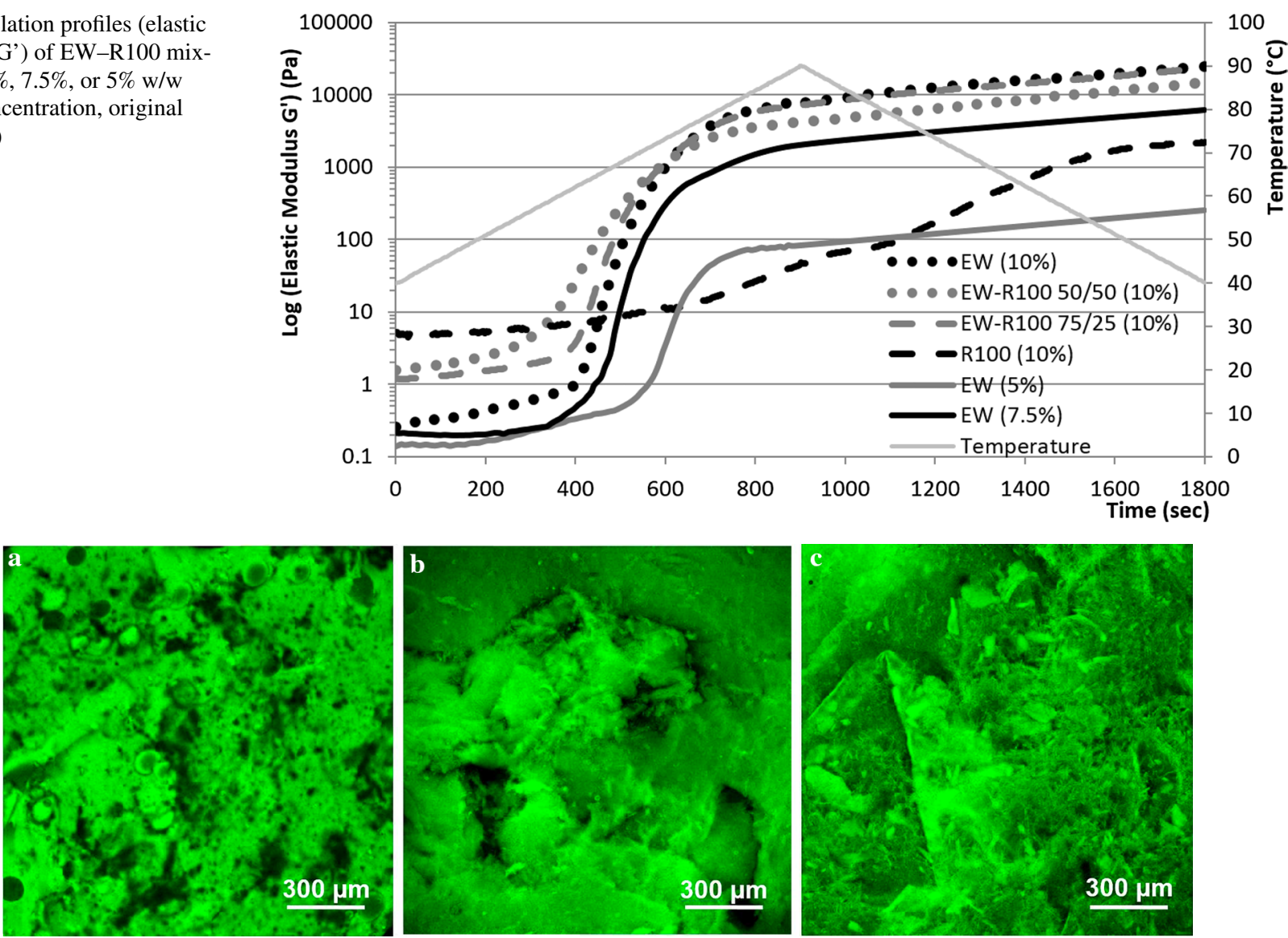

Fig. 5 Confocal microscopy of EW, R100, and 75/25 EW-R100 gels (10\% w/w NCM concentration, original pH, rhodamine, and magnification $\times 10)$. a EW, b 75/25 EW-R100, and c R100 
their meat-like texture and described as a fibre gel composite composed of an entangled mass of mycoprotein hyphae with gelled albumen protein within the interstitial space [6]. EW gels presented a dense homogenous network (Fig. 5a), which correlated with their high viscoelasticity.

R100 gels proved less viscoelastic than 7.5\% EW ones; however, 75/25 EW-R100 gels displayed a higher viscoelasticity than $7.5 \%$ EW ones (Fig. 4), highlighting a synergistic interaction between the R100 material and egg albumen. As reported by Finnigan [6], the mixing of mycoprotein biomass and egg albumen during the production of Quorn mince and pieces introduces laminations which can be considered as textural precursors for the final meat-like texture. In the current study, such interaction could be due to the binding of lysozyme to chitin and chitosan contained in fungal cell walls $[25,26]$, which was supported by the SDS-PAGE results with the respective disappearance and weakening of the dimeric and monomeric lysozyme bands when EW was mixed with R100 (Fig. 2).

In parallel, our previous study [13] showed the presence of compounds with known gelling properties in the centrate, including cell wall and membrane constituents which concentrated in the R100 fraction as part of the hyphal aggregates and contributed to the EW-R100 gelling profile. The gelifiers reported included chitin and chitosan [28, 29], phytosterols and phytosterol esters [34], ceramides and sphingomyelins [35], glycosphingolipids [36], inulin [30], galactan [31], and nucleobases, nucleosides, and nucleotides (including guanine-based compounds) and their derivatives [32].

R100 hydrogels displayed low hardness (Fig. 6). However, 75/25 EW-R100 hydrogels proved harder than 7.5\% EW ones and replicated the hardness of $10 \%$ EW ones, which, in addition to their similar viscoelasticity, highlighted the potential of R100 as partial EW replacer for gelling applications. The 50/50 EW-R100 and 5\% EW hydrogels showed lower and similar hardness values $(9.1 \mathrm{~N}$ for $75 / 25$ EW-R100 and $8.3 \mathrm{~N}$ for $5 \% \mathrm{EW}$, results not shown).

The combined rheological and textural results also highlighted the possibility of re-introducing centrate extracts

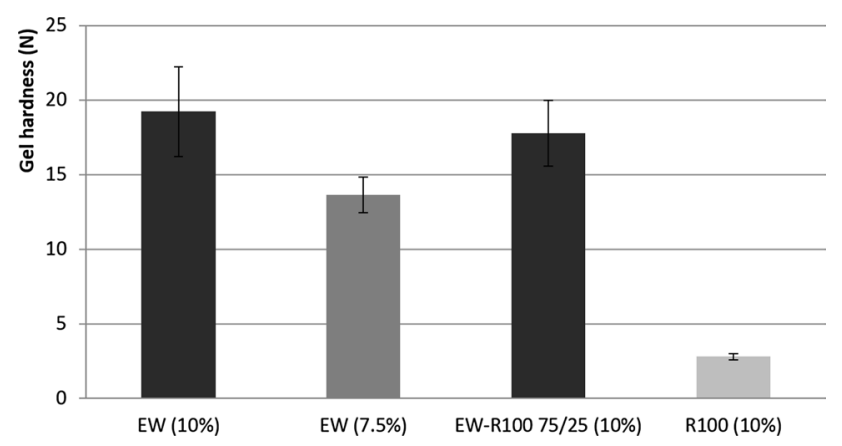

Fig. 6 Hardness profile of EW, R100, and EW-R100 hydrogels (10\% or $7.5 \% \mathrm{w} / \mathrm{w}$ NCM concentration, original $\mathrm{pH}, n=3$ ) back into the process at the manufacturing stage as partial egg albumen replacers while maintaining the characteristic fibre gel composite structure of Quorn products.

\section{Foaming properties of EW-R100 mixtures}

Foams produced by gas sparging with $\mathrm{R} 100$ and 50/50 EW-R100 proved as stable as $1 \% \mathrm{EW}$ foams (foams prepared with $1 \%$ w/w NCM EW solutions) and more stable than $0.5 \%$ EW foams (Fig. 7). Frothing results showed higher foaming abilities for $1 \%$ and $0.75 \% \mathrm{EW}$ in comparison with R100, while 75/25 EW-R100 ranged as intermediate (Fig. 8). R100 foams produced by frothing showed the lowest stability; however, 75/25 EW-R100 foams proved more stable than $0.75 \% \mathrm{EW}$ ones and displayed a similar foam height to $1 \% \mathrm{EW}$ ones after $540 \mathrm{~min}$, indicating a functional synergy between the R100 material and EW proteins.

A high density of air bubbles was observed in EW and 75/25 EW-R100 foams following frothing (Fig. 9a, b) while only few air bubbles were observed in R100 foams

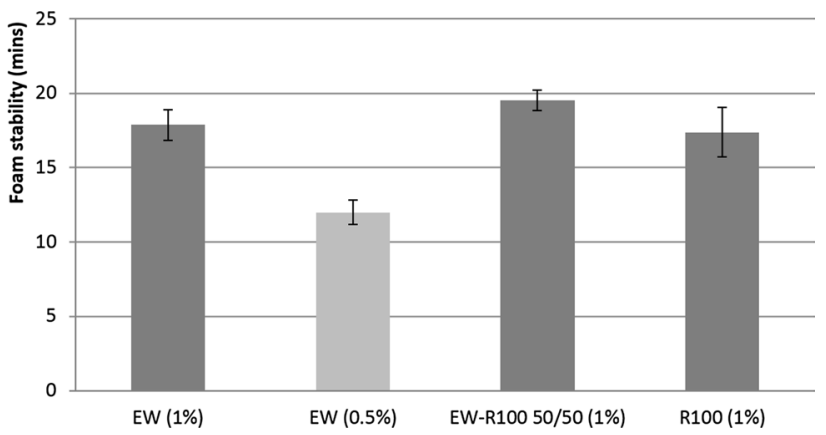

Fig. 7 Foam stability of EW, R100, and 50/50 EW-R100 as determined by the Rudin method ( $1 \%$ or $0.5 \%$ w/w NCM concentration, original $\mathrm{pH}, n=3$ )

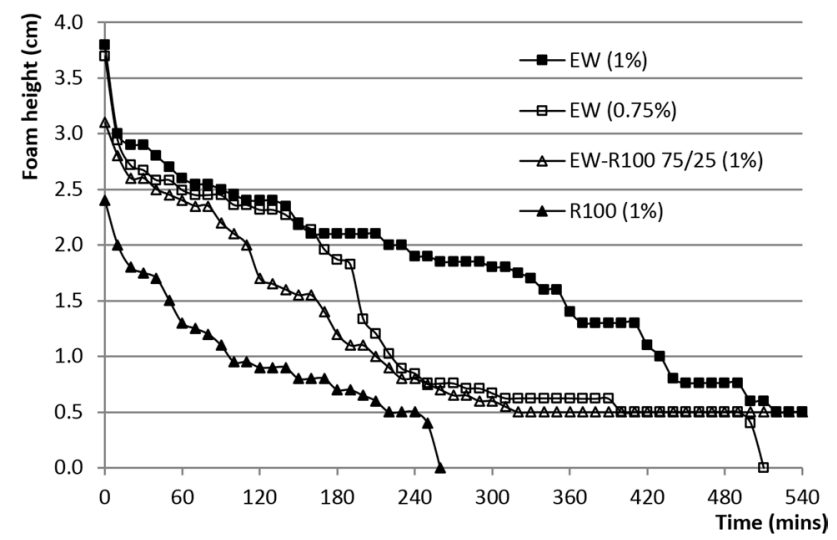

Fig. 8 Foaming ability and stability profiles of EW, R100, and EWR100 mixtures ( $1 \%$ or $0.75 \%$ w/w NCM concentration, original $\mathrm{pH}$, $n=3)$ 

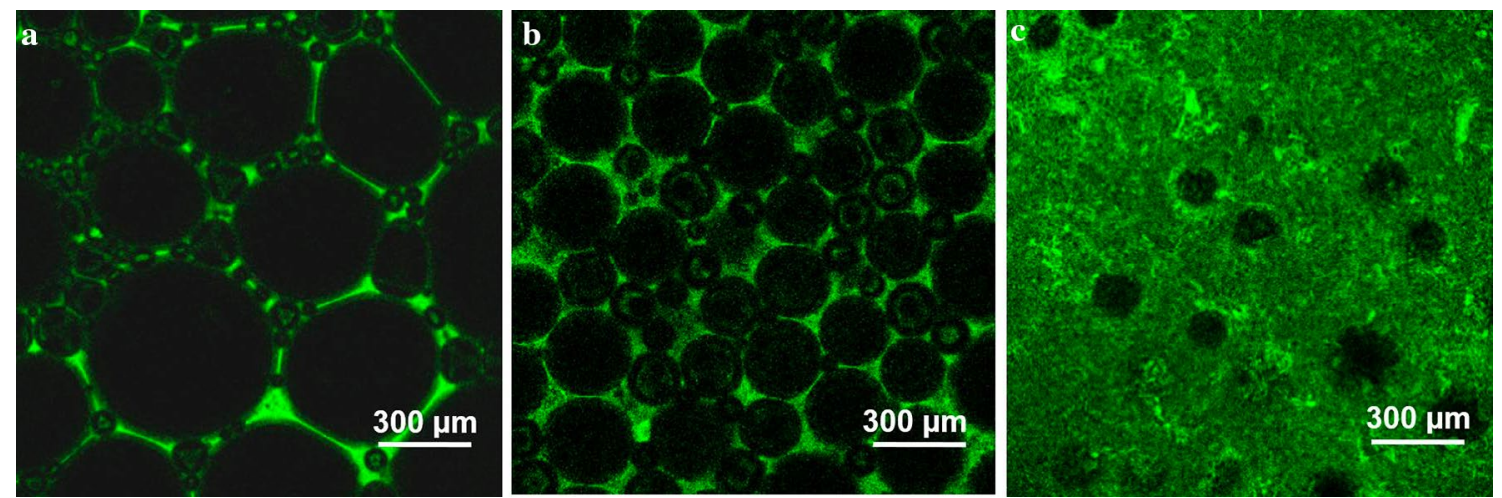

Fig. 9 Confocal microscopy of EW, R100, and 75/25 EW-R100 foams prepared by frothing (1\% w/w NCM concentration, original pH, rhodamine, and magnification $\times 10$ ). a EW, b 75/25 EW-R100, and $\mathbf{c}$ R100

(Fig. 9c). Micrographs showed that air bubbles in R100 foams were trapped in a dense network of hyphae and cell debris (Fig. 9c). These observations were in agreement with previous studies in which highly viscous fermentation media displayed issues with gas-liquid mass transfer for the filamentous fungus Aspergillus terreus [27]. Hence, the high viscosity related to the dense hyphal network observed in R100 solutions (Fig. 1) only allowed a limited number of air bubbles to be formed and transported within the liquid, leading to a poor foaming ability (Fig. 8), but, on the other hand, prevented the destabilisation of the air bubbles formed by limiting their movement in the liquid, leading to a high foam stability (Figs. 7, 8). Foams prepared with 75/25 EW-R100 showed a less dense hyphal network around the air bubbles (Fig. 9b), which, in addition to the action of egg white proteins, allowed a higher number of air bubbles to be formed, leading to a higher foaming ability than R100, while nonetheless contributing to their stabilisation due to viscosity.

In addition, depending on their hydrophobicity, the possible concentration of Fusarium venenatum cells and/or spores in the $\mathrm{R} 100$ fraction as a result of the ultrafiltration process could also have contributed to the stabilisation of R100 and EW-R100 foams as the shapes and sizes of bacterial cells, viruses, and spores fall within the range for stabilisation of biphasic dispersions including foams and emulsions [37]. Moreover, a correlation between foam stability and cell surface hydrophobicity was reported for different strains of Acinetobacter calcoaceticus [38].

In parallel, surface tension measurements were undertaken to assess the potential contribution of surfaceactive material from R100 to the foaming properties of EW-R100 samples. Solutions prepared with $1 \% \mathrm{w} / \mathrm{w}$ NCM EW, R100, and 75/25 EW-R100 showed significantly lower surface tension values than deionised water $(72 \mathrm{mN} / \mathrm{m}$ ) (Fig. 10), which was in agreement with their foaming properties. Measurements were carried out $1 \mathrm{~min}$ after adding the solution to the cell to allow for the large

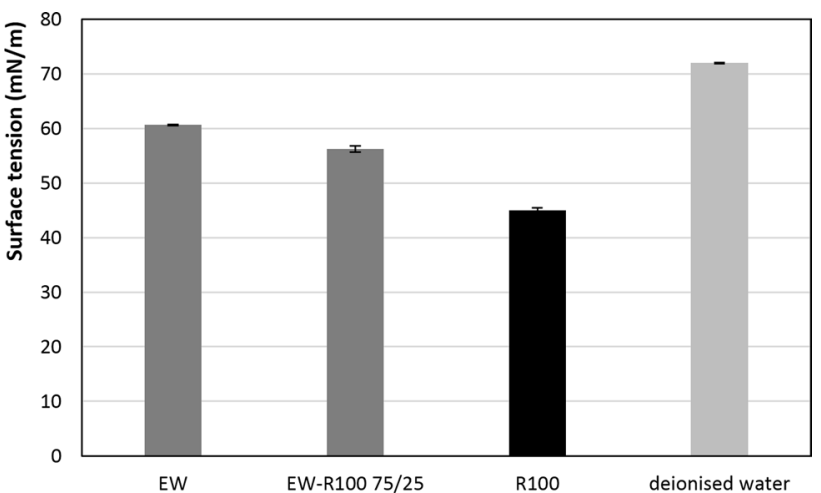

Fig. 10 Surface tension of EW, R100, and 75/25 EW-R100 solutions $(1 \% \mathrm{w} / \mathrm{w} \mathrm{NCM}$ concentration, original $\mathrm{pH}, n=3)$

hyphal structures observed in Fig. $1 \mathrm{~b}$ and $\mathrm{c}$ to settle at the base of the cell, as observed in our previous study using a Krüss Easydrop tensiometer (Krüss GmbH, Germany) equipped with a video camera [13]. The surface tension of R100 and EW-R100 proved lower than that of EW, which highlighted the presence of surface-active molecules from R100 at the air-water interface and their contribution to the foaming properties of EW-R100 samples. We previously reported a number of proteins in the centrate and R100 fraction with known foaming properties [13]. A cerato-platanin was concentrated in the R100 fraction in comparison with the centrate and the serpin SERPB1 was detected in both samples. The cerato-platanin EPL1 from the fungus Trichoderma atroviride was previously reported to form ordered self-assembled layers at the air/ water interface [39] and hydrophobic/hydrophilic interfaces [40], while serpins Z4 and Z7 from barley have been characterised as foam-positive proteins in beer [41, 42]. In addition, a range of metabolites with known foaming properties were also identified in the centrate, including cell wall and membrane constituents which concentrated 
in the R100 fraction as part of the hyphal aggregates and contributed to the EW-R100 foaming profile. The foampositive compounds reported included chitin and chitosan [28, 29], monoglycerides and diglycerides [43], glycosyl diglycerides [44], triterpenoid and steroidal saponins [45], cerato-platanin [46], and fatty amides [47]. Moreover, the possible release of surface-active molecules and cell debris from the R100 hyphal aggregates during gas sparging or frothing could also have contributed to the stabilisation of R100 and EW-R100 foams.

The higher foam stability exhibited by 75/25 EW-R100 in comparison with $0.75 \% \mathrm{EW}$ following frothing (Fig. 8) could also be due to the presence of surface-active aggregates formed between lysozyme and R100 proteins, as suggested by the respective weakening and disappearance of monomeric and dimeric lysozyme bands on the 75/25 EW-R100 SDS-PAGE profile (Fig. 2). Due to its high isoelectric point (10.7), lysozyme was charged positively in the 75/25 EW-R100 mixture and possibly combined with negatively charged proteins present in R100 through electrostatic interactions. Such aggregation process was previously reported between lysozyme and negatively charged whey proteins [11, 12].

Similarly, a synergy in adsorption at the air/water interface between lysozyme and negatively charged R100 proteins when foamed together could also have contributed to the higher foam stability exhibited by $75 / 25 \mathrm{EW}-\mathrm{R} 100$ in comparison with $0.75 \% \mathrm{EW}$ following frothing (Fig. 8) and by 50/50 EW-R100 in comparison with $0.5 \%$ EW following gas sparging (Fig. 7). In this case, intermolecular interactions between the oppositely charged proteins occur at the interface after the unfolding of the proteins, which stabilises the air bubbles due to the reduction of electrostatic repulsive interactions in the protein film. Such synergy at the air/water interface was previously reported between the two egg white proteins lysozyme and ovalbumin [8]. Synergistic effects between lysozyme and whey proteins were shown to result from intermolecular interactions in the bulk solution as well as at the air-water interface, resulting in higher foam stability [11, 12].

The combined foaming, microscopy, and tensiometry results highlighted that surface-active material present in R100 and/or synergistically combining with EW proteins contributed to the high stability of EW-R100 foams, while the dense hyphal network observed in these foams contributed via physical stabilisation of the air bubbles. Foam-positive material present in R100 included hyphal aggregates, fungal cells, foaming molecules, and hyphal fragments or molecules released from hyphal aggregates during the foaming process. These results highlighted the potential of R100 for use as partial replacer of EW as foaming agent.

\section{Conclusions}

The study investigated if a similar synergy to the one reported between EW and mycoprotein in the formation of the Quorn meat-like texture could be exploited between EW and a functional extract from the Quorn fermentation coproduct (R100). The viscoelasticity and hardness of 75/25 EW-R100 hydrogels proved similar to $10 \%$ EW ones and higher than 7.5\% EW ones. Frothed 75/25 EW-R100 foams showed similar stability to $1 \% \mathrm{EW}$ ones and higher stability than $0.75 \%$ EW ones, while gas-sparged 50/50 EW-R100 foams proved as stable as $1 \% \mathrm{EW}$ ones and more stable than $0.5 \% \mathrm{EW}$ ones. These results highlighted the potential of centrate extracts for partial EW replacement as foaming and gelling agent.

R100 foams prepared by frothing proved less stable than 7.5\% EW ones; however, a 75/25 w/w EW-R100 mixture displayed a higher foam stability than $7.5 \%$ EW ones. Similarly, R100 gels proved less viscoelastic than $7.5 \%$ EW ones; however, 75/25 EW-R100 gels displayed a higher viscoelasticity than $7.5 \% \mathrm{EW}$ ones. Both results highlighted a functional synergy between the R100 material and EW proteins.

In parallel tensiometry measurements highlighted the presence of surface-active material in EW-R100 mixtures contributing to their high foaming properties. In particular, the SDS-PAGE profile of EW-R100 mixtures indicated a possible aggregation of monomeric and dimeric forms of lysozyme with R100 proteins and/or fungal cells, contributing to their foaming and rheological properties. The results highlighted the complex nature of the functional profile of EW-R100 mixtures, with contributions reported for both hyphal structures and surface-active material.

Future work will assess the partial functional replacement of EW ingredients by centrate extracts in food formulations. Additional studies are needed to further understand the potential synergy between EW and R100 and the contribution of both hyphal structures and surface-active molecules to EW-R100 functionality. Finally, this study highlights the possibility of re-introducing Quorn fermentation co-product extracts back into the process at the manufacturing stage as partial egg albumen replacers.

Acknowledgements The authors wish to thank Dr. Tim Finnigan and Dr. Sue Gordon from Marlow Foods for their support during this project.

Funding This work was supported by the Engineering and Physical Sciences Research Council [Grant Number EP/J501682/1 Foaming and Fat Replacer Ingredients].

\section{Compliance with ethical standards}

Conflict of interest The authors declare that they have no conflict of interest. 
Compliance with ethics requirements This article does not contain any studies with human or animal subjects.

Open Access This article is distributed under the terms of the Creative Commons Attribution 4.0 International License (http://creativecommons.org/licenses/by/4.0/), which permits unrestricted use, distribution, and reproduction in any medium, provided you give appropriate credit to the original author(s) and the source, provide a link to the Creative Commons license, and indicate if changes were made.

\section{References}

1. McMichael J, Powles C, Butler R (2007) Food, livestock production, energy, climate change, and health. Lancet 370:1253-1263

2. Denny A, Aisbitt B, Lunn J (2008) Mycoprotein and health. Br Nutr Bull 33:298-310

3. Edelman J, Fewell A, Solomons GL (1983) Myco-protein-a new food. Nutr Abstr Rev Clin Nutr 53:471-480

4. Finnigan TJA, Lemon M, Allen B, Patton I (2010) Mycoprotein LCA and food 2030. Asp Appl Biol 102:81-90

5. Ward PN (1996) A process for the reduction of nucleic acid content for a fungus imperfectus. WO Patent 95/23843

6. Finnigan T (2011) Mycoprotein: origins, production and properties. In: Phillips GO, Williams PA (eds) Handbook of food proteins. Woodhead Publishing, Sawston

7. Lechevalier V, Croguennec T, Pezennec S, Guérin-Dubiard C, Pasco M, Nau F (2003) Ovalbumin, ovotransferrin, lysozyme: three model proteins for structural modifications at the air-water interface. J Agric Food Chem 51:6354-6361

8. Floch-Fouéré C, Pezennec S, Lechevalier V, Beaufils S, Desbat B, Pezolet M, Renault A (2009) Synergy between ovalbumin and lysozyme leads to non-additive interfacial and foaming properties of mixtures. Food Hydrocoll 23(2):352-365

9. Lechevalier V, Périnel E, Jeanete R, Lesaffre C, Croguennec T, Guérin-Dubiard C, Pasco M, Nau F (2005) Evidence for synergy in the denaturation at the air-water interface of ovalbumin, ovotransferrin and lysozyme in ternary mixture. Food Chem 92:79-87

10. Domadaran S, Anand K, Razumovsky L (1998) Competitive adsorption of egg white proteins at the air-water interface: direct evidence for electrostatic complex formation between lysozyme and other egg proteins at the interface. J Agric Food Chem 46:872-876

11. Howell NK, Yeboah NA, Lewis DFV (1995) Studies on the electrostatic interactions of lysozyme with alpha-lactalbumin and beta-lactoglobulin. Int J Food Sci Technol 30(6):813-824

12. Kuropatwa M, Tolkach A, Kulozik U (2009) Impact of pH on the interaction between whey and egg white proteins as assessed by foamability of their mixtures. Food Hydrocoll 23:2174-2181

13. Lonchamp J, Clegg PS, Euston SR (2019) Foaming, emulsifying and rheological properties of extracts from a co-product of the Quorn fermentation process. Eur Food Res Technol. https://doi. org/10.1007/s00217-019-03287-z

14. Lynch JM, Barbano DM, Fleming JR (1998) Indirect and direct determination of the casein content of milk by Kjeldahl nitrogen analysis: collaborative study. J AOAC Int 81:763-774

15. Sadler MJ (2003) Mycoprotein. In: Caballero B, Trugo M, Finglas PM (eds) Encyclopedia of food sciences and nutrition (second edition). Academic Press, Cambridge

16. Laemmli UK (1970) Cleavage of structural proteins during the assembly of the head of bacteriophage T4. Nature 227:680-685
17. Havea P, Watkinson P, Kuhn-Sherlock B (2009) Heat-induced whey protein gels: protein-protein interactions and functional properties. J Agric Food Chem 57:1506-1512

18. Rudin AD (1957) Measurement of the foam stability of beers. $\mathrm{J}$ Inst Brew 63(6):506-509

19. Zhang J, Zhang J (2016) The filamentous fungal pellet and forces driving its formation. Crit Rev Biotechnol 36(6):1066-1077

20. Nyman J, Lacintra MJ, Westman JO, Berglin M, Lundin M, Lennartsson PR, Taherzadeh MJ (2013) Pellet formation of zygomycetes and immobilization of yeast. New Biotechnol 30(5):516-522

21. Onuma K, Inaka K (2008) Lysozyme dimer association: similarities and differences compared with lysozyme monomer association. J Cryst Growth 310(6):1174-1181

22. Desfougères $Y$, Lechevalier V, Pezennec S, Artzner F, Nau F (2008) Dry-heating makes hen egg white lysozyme an efficient foaming agent and enables its bulk aggregation. J Agric Food Chem 56(13):5120-5128

23. Narai-Kanayama A, Hanaishi T, Aso K (2016) Mechanistic investigation of capability of enzymatically synthesized polycysteine to cross-link proteins. Biochem Biophys Rep 7:338-346

24. McKenzie HA, White FH (1991) Lysozyme and alpha-lactalbumin: structure, function, and interrelationships. Adv Protein Chem 41:173-315

25. Ruckenstein E, Zeng X (1997) Macroporous chitin affinity membranes for lysozyme separation. Biotechnol Bioeng 56:610-617

26. Nordtveit RJ, Vårum KM, Smidsrød O (1994) Degradation of fully water-soluble, partially $N$-acetylated chitosans with lysozyme. Carbohydr Polym 23:253-260

27. Porcel EMR, Casas Lopez JL, Sanchez Perez JA, Fernandez Sevilla JM, Chisti Y (2005) Effects of pellet morphology on broth rheology in fermentations of Aspergillus terreus. Biochem Eng J 26:139-144

28. Lapasin R, Stefancic S, Delben F (1996) Rheological properties of emulsions containing soluble chitosan. Agro Food Ind High Tech 7:12-17

29. Quintela S, Villarán MC, López De Armentia I, Elejalde E (2012) Ochratoxin a removal from red wine by several oenological fining agents: bentonite, egg albumin, allergen-free adsorbents, chitin and chitosan. Food Addit Contam Part A 29(7):1168-1174

30. Mensink MA, Frijlink HW, van der Voort Maarschalk K, Hinrichs WLJ (2015) Inulin, a flexible oligosaccharide I: review of its physicochemical characteristics. Carbohydr Polym 130:405-419

31. Delattre C, Fenoradosoa TA, Michaud P (2011) Galactans: an overview of their most important sourcing and applications as natural polysaccharides. Braz Arch Biol Technol 54(6):1075-1092

32. Peters GM, Davis JT (2016) Supramolecular gels made from nucleobase, nucleoside and nucleotide analogs. Chem Soc Rev 45:3188-3206

33. Zhu C, Ma Y, Zhou C (2010) Densities and viscosities of sugar alcohol aqueous solutions. J Chem Eng Data 55(9):3882-3885

34. Matheson A, Dalkas G, Clegg PS, Euston SR (2018) Phytosterolbased edible oleogels: a novel way of replacing saturated fat in food. Nutr Bull 43(2):189-194

35. Castro BM, de Almeida RF, Silva LC, Fedorov A, Prieto M (2007) Formation of ceramide/sphingomyelin gel domains in the presence of an unsaturated phospholipid: a quantitative multiprobe approach. Biophy J 93(5):1639-1650

36. Westerlund B, Slotte JP (2009) How the molecular features of glycosphingolipids affect domain formation in fluid membranes. Biochimica et Biophysica Acta (BBA)-Biomembranes 1788(1):194-201

37. Lam S, Velikov KP, Velev OD (2014) Pickering stabilization of foams and emulsions with particles of biological origin. Curr Opin Colloid Interface Sci 19(5):490-500 
38. Rosenberg M, Rosenberg E (1985) Bacterial adherence at the hydrocarbon-water interface. Oil Petrochem Pollut 2(3):155-162

39. Frischmann A, Neudl S, Gaderer R, Bonazza K, Zach S, Gruber S, Spadiut O, Friedbacher G, Grothe H, Seidl-Seiboth V (2013) Self-assembly at air/water interfaces and carbohydrate binding properties of the small secreted protein EPL1 from the fungus Trichoderma atroviride. J Biol Chem 288:4278-4287

40. Bonazza K, Gaderer R, Neudl S, Przylucka A, Allmaier G, Druzhinina IS, Grothe H, Friedbacher G, Seidl-Seiboth V (2015) The fungal cerato-platanin protein EPL1 forms highly ordered layers at hydrophobic/hydrophilic interfaces. Soft Matter 11(9):1723-1732

41. Li X, Jin Z, Gao F, Lu J, Cai G, Dong J, Yu J, Yang M (2014) Characterization of barley serpin $\mathrm{Z7}$ that plays multiple roles in malt and beer. J Agric Food Chem 62(24):5643-5650

42. Specker C, Niessen L, Vogel RF (2014) In vitro studies on the main beer protein $\mathrm{Z} 4$ of Hordeum vulgare concerning heat stability, protease inhibition and gushing. J Inst Brew 120:85-92

43. Moonen H, Bas H (2004) Mono- and diglycerides. In: Whitehurst RJ (ed) Emulsifiers in food technology. Blackwell Publishing Ltd, Oxford
44. Keller RCA, Orsel R, Hamer RJ (1997) Competitive adsorption behaviour of wheat flour components and emulsifiers at an airwater interface. J Cereal Sci 25:175-183

45. Kharkwal H, Panthari P, Pant MK, Kharkwal H, Kharkwal AC, Joshi DD (2012) Foaming glycosides: a review. IOSR J Pharm 2(5):23-28

46. Al-Ahmad K (2015) The definition, preparation and application of rhamnolipids as biosurfactants. Int J Nutr Food Sci 4(6):613-623

47. Xu W, Gu H, Zhu X, Zhong Y, Jiang L, Xu M, Song A, Hao J (2015) $\mathrm{CO}_{2}$-controllable foaming and emulsification properties of the stearic acid soap systems. Langmuir 31(21):5758-5766

Publisher's Note Springer Nature remains neutral with regard to jurisdictional claims in published maps and institutional affiliations. 